\title{
ANÁLISE DA INFLUÊNCIA DO \\ CONFORMAL COOLING EM UM MOLDE DE INJEÇÃO DE PLÁSTICO COMPARADO COM A REFRIGERAÇÃO CONVENCIONAL POR SIMULAÇÃO DE TRANSFERÊNCIA DE CALOR
}

Adriano Fagali de Souza ${ }^{1}$ Rafael Felipe Fronza Santos ${ }^{2}$ Sabrina Marques ${ }^{3}$

\section{RESUMO}

$\mathrm{Na}$ injeção de peças plásticas, melhorar a qualidade do produto e reduzir o tempo do ciclo de injeção é o foco em todas as fases do projeto de um molde de injeção. Durante o processo de injeção, a fase de resfriamento temo impacto mais significativo sobre o tempo total do ciclo, além de influenciar diretamente as propriedades das peças injetadas. Assim, o resfriamento das peças deve ser tão uniforme e rápido quanto possível. Para atingir este objetivo, os canais de refrigeração nas cavidades devem ser projetados próximos da geometria do produto. No entanto, os métodos convencionais para a fabricação de canais de refrigeração permitem produzir apenas orifícios lineares. A Fusão Seletiva a Laser (SLM) é uma tecnologia de Manufatura Aditiva à base de pó metálico capaz de produzir peças camada por camada, diretamente a partir do modelo CAD. Pode ser utilizada para a fabricação de insertos para injeção de plásticos com canais de refrigeração complexos, conhecidos como Conformal Cooling. Este estudo investigou, por simulação durante o processo de injeção para produzir peças em polipropileno, o efeito do sistema de refrigeração Conformal Cooling. Projetou-se para o mesmo produto inserto superior e inferior com sistema de refrigeração convencional e com sistema Conformal Cooling. Os resultados indicam que o sistema Conformal Cooling, em comparação com o sistema de refrigeração convencional, pode fornecer transferência de calor homogênea durante o processo de resfriamento e proporcionar uma redução do tempo total do ciclo de aproximadamente $14 \%$.

Palavras-Chave: Simulação. Injeção de plástico. Conformal Cooling.

Manufatura aditiva. SLM - Fusão seletiva a laser. 


\section{INTRODUÇÃO}

As indústrias de transformação de plásticos se comportam como uma força motriz para o mercado atual. Tal processo de fabricação requer conhecimento de ferramental, como moldes para a fabricação de peças plásticas. (SOUZA et al., 2010; SANT'ANNA; Wiebeck, 2006).

\section{O MOLDE É O COMPONENTE}

MAIS IMPORTANTE NO CICLO DE

INJEÇÃO DE UMA PEÇA PLÁSTI-

CA, POIS O SEU DESEMPENHO

TÉRMICO INflUENCIA NO TEMPO

DE CICLO E NA QUALIDADE DO

PRODUTO.

Conforme Duleba e Greskovic (2012), as propriedades ideais de peças plásticas podem ser encontradas somente quando a temperatura do molde definida é mantida durante o processo. A temperatura do molde afeta as propriedades mecânicas, o comportamento de contração, empenamento, qualidade superficial, tempo de ciclo e comprimento de fluxo em peças com paredes finas. A fase de resfriamento durante um processo de injeção representa normalmente aproximadamente $2 / 3$ do tempo total de ciclo.

Atualmente, o sistema de refrigeração dentro das cavidades do molde é fabricado por meio da remoção de material pelo método de furação. Isso significa que somente canais lineares são possíveis de fabricar e, na maioria dos casos, a transferência de calor uniforme não é possível obter.Novas tecnologias para fabricar peças metálicas têm sido desenvolvidas na última década, tal como impressão metálica 3D e processos de sinterização. Essas técnicas são conhecidas como Manufatura Aditiva (AM) e permitem fabricar peças com geometria livre e elas têm sido utilizadas com sucesso na indústria médica e aeroespacial. (WOHLERS, 2012; DIMLA et al., 2005; LI et al., 2005; SILVA, 2009).

Dentre as técnicas de AM, a Fusão Seletiva a Laser (SLM) é a única tecnologia a base de pó metálico para produzir objetos com geometria complexa e propriedades mecânicas comparadas às do material a granel (laminado ou trefilado) (YADROITSEV,2010;2011).A técnica SLM pode ser aplicada para fabricar moldes com sistema de refrigeração Conformal Cooling. No entanto, o custo para utilizar essa tecnologia é ainda muito alto. Então, os benefícios do Conformal Cooling para a injeção de plástico precisam ser muito claros para conhecer a viabilidade da fabricação de um molde por SLM.

De acordo com Dalgarno e Stewart (2001), a utilização dos canais Conformal Cooling em ferramentais para moldes de injeção podem oferecer redução significativa no tempo de ciclo. Ilyas et al. (2010) identificaram uma melhoria na produtividade e economia de energia devido à elevada eficiência na transferência de calor fornecida pelos canais em Conformal Cooling. Hsu et al. (2012) realizaram simulações tridimensionais e concluíram que o Conformal Cooling é eficiente na redução do tempo de refrigeração. No entanto, os estudos citados foram desenvolvidos nos Estados Unidos e na Europa. No Brasil, tanto a tecnologia Conformal Cooling como o processo SLM, ainda são muito pouco divulgados e praticamente não existe conhecimento sobre o assunto. 
Nesse contexto, o presente trabalho tem como objetivo investigar a influência do sistema de refrigeração Conformal Cooling por simulação de elementos finitos (CAE) na transferência de calor durante o processo de injeção e as características do ciclo para produzir uma determinada peça em polipropileno (PP).

\section{ANÁLISE TÉRMICA}

O calor conduzido no interior das cavidades do molde pelo material fundido necessita ser extraído, assegurando a solidificação do fundido, fazendo com que ele assuma o perfil da cavidade do molde e, após, permita a ação do sistema de extração para o produto moldado, sem danos. (BRITO et al., 2004).

No geral, o material polimérico na saída da máquina injetora está a uma temperatura de aproximadamente $230^{\circ} \mathrm{C}$ e o molde a $30^{\circ} \mathrm{C}$. A troca de calor ocorre entre o molde e o material fundido, causando um constante aumento da temperatura do molde até sua estabilização. (SACCHELLI; CARDOSO, 2013).

De acordo com Park e Dang (2012), quando a troca de calor é estabilizada, o fluxo de calor realizado para o molde e o fluxo de calor removido do molde estão em equilíbrio. A troca de calor é apresentada pela equação 1 :

$$
Q_{m}+Q_{c}+Q_{e}=0
$$

onde $Q_{m}, Q_{c}$ e $Q_{e}$ são respectivamente: a troca de calor com o fundido, a troca de calor com a refrigeração e a troca de calor com o ambiente.

\subsection{Tempo de resfriamento}

O tempo de resfriamento é proporcional ao quadrado da maior espessura da parede da peça mais o maior diâmetro do canal de refrigeração elevado à potência de 1,6, e inversamente proporcional à difusividade térmica do polímero fundido. (DULEBA; GRESKOVIC, 2012).

Essas relações são dadas pela equação 2:

$$
T_{c} \propto\left(T h_{w}^{2}+D_{r}^{1.6}\right) \propto \frac{1}{a}
$$

onde $T_{c}$ é o tempo de resfriamento $T h_{w}^{2}$, é a maior espessura da parede da peça, $D_{r}$ é o maior diâmetro do canal de refrigeração, e $a$ é a difusividade térmica do polímero fundido.

A difusividade térmica do polímero fundido é definida de acordo com a equação 3 (VENERUS et al., 1999):

$$
a=\frac{K}{\rho C_{v}}
$$

onde $K$ é a condutividade térmica, $\rho$ é a densidade, e $C_{v}$ é o calor específico a volume constante. 
O número de Reynolds é descrito de acordo com a equação 4 (DULEBA; GRESKOVIC, 2012):

$$
R e=\frac{\rho U d}{\eta}
$$

onde $\rho$ é a densidade dolíquido refrigerante, $U$ é a média da velocidade do líquido refrigerante, $d$ é o diâmetro do canal da refrigeração, e $\eta$ é a viscosidade dinâmica do refrigerante. $\mathrm{O}$ tipo de fluxo do refrigerante pode ser determinado pelo número de Reynolds $R e$, como mostra a tabela 1:
Tabela 1: Tipo de fluxo do refrigerante

\begin{tabular}{|l|l|}
\hline Número de Reynolds, $\left(R_{e}\right)$ & Tipo de fluxo \\
\hline $4000<\left(R_{e}\right)$ & Turbulento \\
\hline $2300<\left(R_{e}\right)<4000$ & Transitório \\
\hline $100<\left(R_{e}\right)<2300$ & Laminar \\
\hline$\left(R_{e}\right)<100$ & Estagnado \\
\hline
\end{tabular}

Fonte: Duleba e Greskovic (2012)

\section{MATERIAIS E MÉTODOS}

Um porta-ovos para refrigerador foi escolhido como workpiece devido à complexidade da geometria. A figura 1 apresenta a workpiece, as cavidades e o molde de injeção utilizado neste estudo.

Figura 1: Workpiece, cavidades e molde de injeção
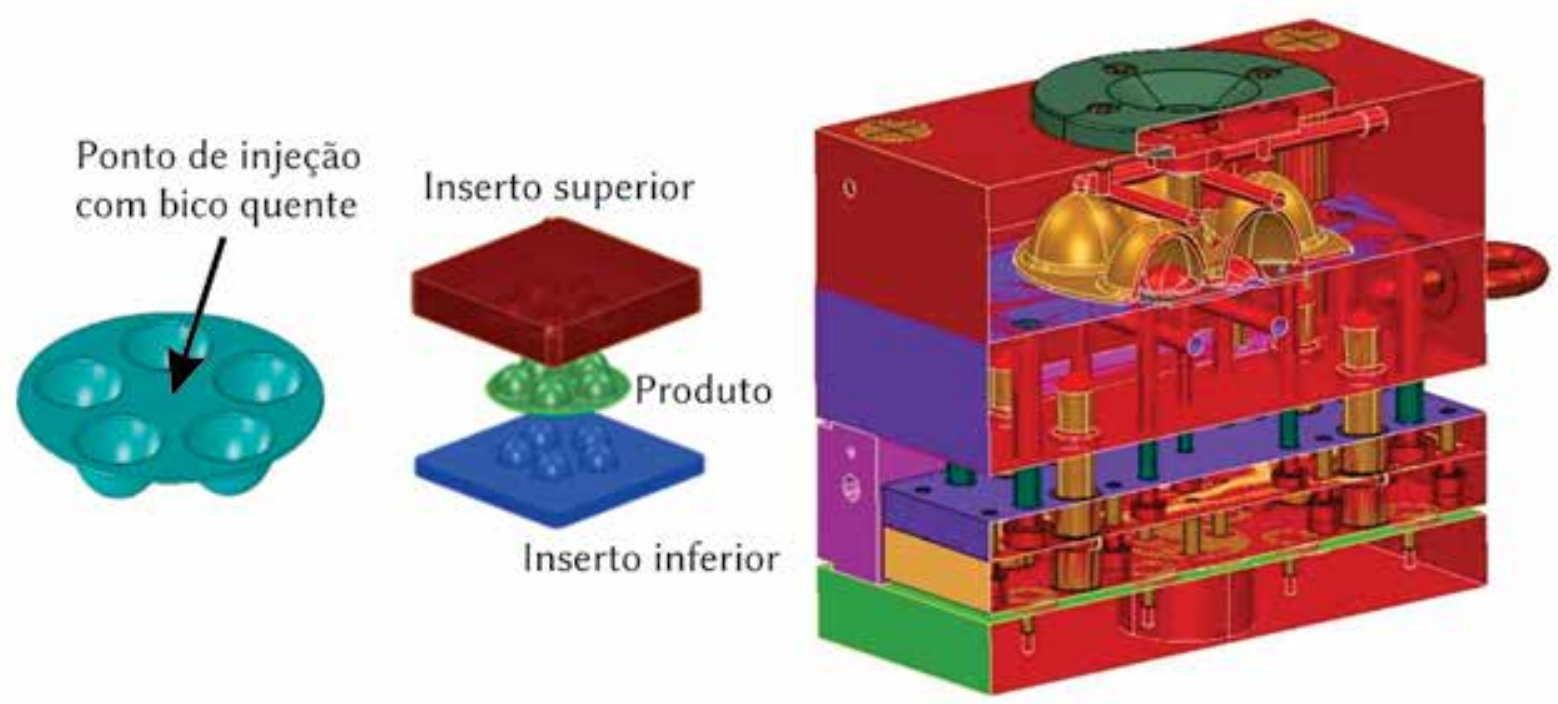

Fonte: Souza e Bodziak (2012) 
Ageometria da workpiece possui cinco cavidades conectadas por uma espessura de $2 \mathrm{~mm}$ e um diâmetro total de $140 \mathrm{~mm}$. As dimensões da cavidade superior são $190 \mathrm{~mm}$ X $155 \mathrm{~mm} \mathrm{X}$ $65 \mathrm{~mm}$ e as dimensões da cavidade inferior são $190 \mathrm{~mm}$ X $155 \mathrm{~mm}$ X $61 \mathrm{~mm}$.

O material utilizado no processo de injeção foi o polipropileno H 201 fornecido pela empresa Braskem (tabela 2) e os dados de entrada do processo foram: material das cavidades INOX-420; tipo de injeção - com bico quente diretamente na peça; temperatura de injeção $230^{\circ} \mathrm{C}$; tempo de injeção- $1 \mathrm{~s}$; fluido refrigerante - água (temperatura $25^{\circ} \mathrm{C}$ ); pressão máxima de injeção da máquina - $180 \mathrm{Mpa}$; máxima força de fechamento da máquina - 120 ton. $\mathrm{O}$ software CAE utilizado para a simulação foi o Moldflow $\mathrm{V} 10^{\circledR}$.
Tabela 2: Propriedades físicas e mecânicas do polipropileno (PP)

\begin{tabular}{|c|c|}
\hline Propriedades & PP H 201 \\
\hline Índice de fluidez (g/10min) & 20 \\
\hline Densidade $\left(\mathrm{g} / \mathrm{cm}^{3}\right)$ & 0,905 \\
\hline Módulo de flexão secante $1 \%(\mathrm{GPa})$ & 1,5 \\
\hline $\begin{array}{l}\text { Resistência à tração no escoamento } \\
(\mathrm{MPa})\end{array}$ & 34 \\
\hline Alongamento no escoamento (\%) & 12 \\
\hline Dureza rockwell (Escala $\mathrm{R}$ ) & 102 \\
\hline $\begin{array}{l}\text { Resistência ao impacto Izod a } 23^{\circ} \mathrm{C} \\
(\mathrm{J} / \mathrm{m})\end{array}$ & 23 \\
\hline $\begin{array}{l}\text { Temperatura de deflexão térmica } \\
\text { a } 0,455 \mathrm{MPa}\left({ }^{\circ} \mathrm{C}\right)\end{array}$ & 97 \\
\hline $\begin{array}{l}\text { Temperatura de deflexão térmica } \\
\text { a } 1,820 \mathrm{MPa}\left({ }^{\circ} \mathrm{C}\right)\end{array}$ & 57 \\
\hline $\begin{array}{l}\text { Temperatura de amolecimento } \\
\text { Vicat a } 10 \mathrm{~N}\left({ }^{\circ} \mathrm{C}\right)\end{array}$ & 154 \\
\hline
\end{tabular}

Fonte: Adaptado de Braskem (2009)

\subsection{Projeto dos canais de refrigeração}

Dois projetos de canais de refrigeração foram investigados: i) canais de refrigeração convencional; ii) canais Conformal Cooling, como é detalhado a seguir. O quadro 1 mostra os dois sistemas de refrigeração desenvolvidos para este estudo.

Quadro 1: Cavidade superior e inferior com diferentes projetos de canais de refrigeração

\begin{tabular}{|l|l|l|}
\hline Descrição & Cavidade Superior & Cavidade Inferior \\
\hline Cavidades & & \\
\hline
\end{tabular}




\begin{tabular}{|c|c|}
\hline Descrição & Cavidade Superior \\
Canais de refrigeração \\
convencional (linear)
\end{tabular}

Fonte: Dos autores (2014)

\subsubsection{Projeto do sistema de refrigeração convencional}

O sistema de refrigeração convencional é fabricado por furação e normalmente requer tampões para ligar os canais e para fechar o circuito. $\mathrm{O}$ fluido flui para as cavidades do molde e volta ao ponto de partida. Devido às restrições do presente processo, os circuitos desenvolvidos normalmente não seguem a geometria do produto, visto que são canais lineares. (HARADA, 2004; MANRICH, 2005).

\subsubsection{Projeto do sistema de refrigeração Conformal Cooling}

O projeto do Conformal Cooling está de acordo com os parâmetros mostrados no escopo geral de projeto de canais (figura 2 e tabela 3 ).

Figura 2: Projeto ideal para sistemas de canais em três dimensões
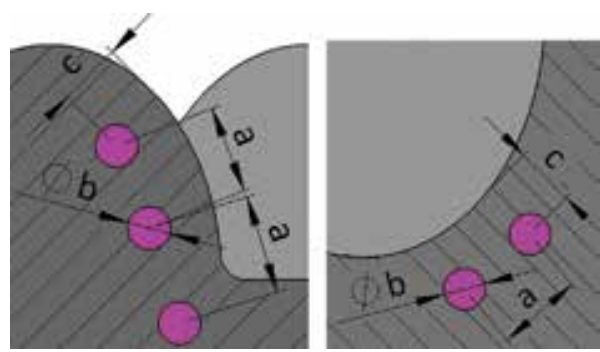

Fonte: Siegfried (2009)

Tabela 3: Dimensões ideais para sistemas de canais

\begin{tabular}{|c|c|c|c|}
\hline $\begin{array}{c}\text { Espessura da parede } \\
\text { do produto moldado } \\
(\mathbf{m m})\end{array}$ & $\begin{array}{c}\text { Diâmetro } \\
\text { do canal, } \\
\mathbf{b}(\mathbf{m m})\end{array}$ & $\begin{array}{c}\text { Distância entre os } \\
\text { centros dos canais, } \\
\mathbf{a}(\mathbf{m m})\end{array}$ & $\begin{array}{c}\text { Distância entre o centro } \\
\text { do canal e a cavidade, } \\
\mathbf{c}(\mathbf{m m})\end{array}$ \\
\hline $0-2$ & $4-8$ & $2-3 \times \mathrm{b}$ & $1.5-2 \times \mathrm{b}$ \\
\hline $2-4$ & $8-12$ & $2-3 \times \mathrm{b}$ & $1.5-2 \times \mathrm{b}$ \\
\hline $4-6$ & $12-14$ & $2-3 \times \mathrm{b}$ & $1.5-2 \times \mathrm{b}$ \\
\hline
\end{tabular}

Fonte: Siegfried (2009) 
De acordo com especificações do projeto, a espessura do produto moldado (workpiece) é 2mm.Logo, as dimensões do sistema Conformal
Cooling foram: b: $5.0 \mathrm{~mm}$, a: $2.5 \times 5.0=12.5 \mathrm{~mm}$, c: $1.7 \times 5.0=8.5 \mathrm{~mm}$.

\section{RESULTADOS E DISCUSSÕES}

Os resultados das simulações são apresentados por gráficos com representações em cores, comparando os dois sistemas de refrigeração investigados. Alguns gráficos tiveram de ser apresentados em escalas individuais, a fim de ter uma boa representação do fenômeno de resfriamento.

Nas figuras 3 e 4 são apresentados a taxa de fluxo do refrigerante e o número de Reynolds nos circuitos de refrigeração, consequentemente.

Figura 3: Taxa de fluxo do líquido refrigerante nos circuitos

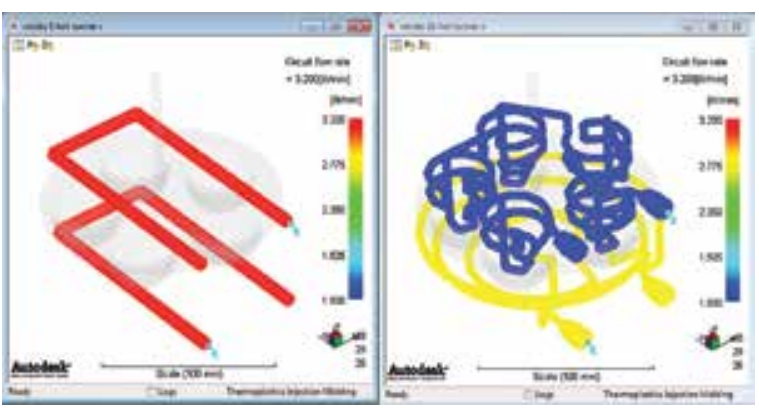

Fonte: Dos autores (2014)

Figura 4: Número de Reynolds nos circuitos

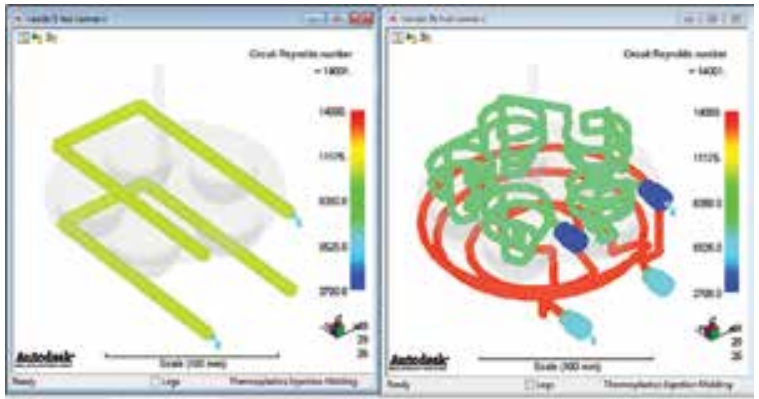

Fonte: Dos autores (2014)
Os resultados da simulação da taxa de fluxo nos circuitos e do número de Reynolds são utilizados para determinar a taxa de fluxo necessária para atingir um fluxo turbulento do líquido refrigerante. Somente a taxa de fluxo não é um fator dominante na extração de calor, mas deve ser o mínimo para atingir o número de Reynolds necessário.

Um número de Reynolds de 4000 ou superior representa um fluxo turbulento, que é preferido em circuitos de refrigeração.

NO ENTANTO, QUANTO MAIOR

O NÚMERO DE REYNOLDS NO

CIRCUITO, MAIOR É A ENERGIA

NECESSÁRIA PARA CIRCULAR O

LÍQUIDO REFRIGERANTE.

Assim, o número de Reynolds ideal para circuitos de refrigeração é de aproximadamente 10.000. As perdas de energia durante a circulação do refrigerante associadas ao número de Reynolds superior a 10.000 superam os ganhos de transferência de calor que podem ser alcançados com números de Reynolds mais elevados. (DULEBA; GRESKOVIC, 2012; REES, 1995; STEIKO, 2004). 
Os resultados apresentados nas figuras anteriores confirmam a teoria de que em circuitos em série a taxa de fluxo e o número de Reynolds são constantes, pois o número de Reynolds não se alterou no decorrer do circuito nas cavidades superior e inferior, tanto para o sistema com canais convencionais como para o sistema com canais Conformal Cooling.

\section{ESSES RESULTADOS MOSTRAM}

QUE NO SISTEMA COM CANAIS

CONVENCIONAIS NAS DUAS

CAVIDADES O NÚMERO DE

REYNOLDS É 10.000 E A TAXA

DE fluXO É DE 3,2 L/MIN.

No sistema com canais Conformal Cooling, o número de Reynolds na cavidade superior é de 12.000 e na cavidade inferior 9.000. A taxa de fluxo apresentou valores de 1,5 1/min para a cavidade superior e $2,81 / \mathrm{min}$ para a cavidade inferior.

As taxas de fluxo apresentadas na figura 3 são suficientes para atingir um fluxo turbulento do líquido refrigerante, pois os valores do número de Reynolds apresentados na figura 4 estão próximos de 10.000 , que é o valor ideal. No entanto, o número de Reynolds apresentado nos circuitos Conformal Cooling é superior ao circuito convencional devido à complexidade geométrica dos canais ser maior, mas os valores ainda estão próximos ao valor ideal.

A figura 5 apresenta a temperatura do líquido refrigerante em todo o circuito de refrigeração. Esta temperatura está relacionada com a taxa de fluxo do refrigerante e com a pressão aplicada nos circuitos.
Figura 5: Temperatura do líquido refrigerante nos circuitos

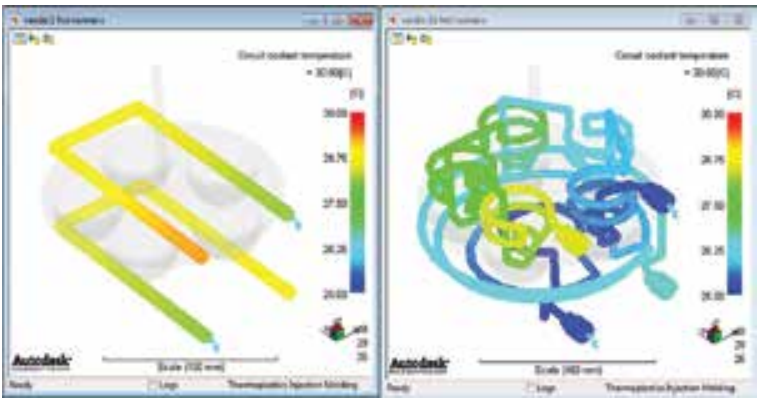

Fonte: Dos autores (2014)

A temperatura do líquido refrigerante varia ao longo do circuito. Como é possível verificar na figura 5, essa variação ocorre linearmente e a temperatura na saída dos circuitos é maior que ao longo do circuito.

A diferença de temperatura do refrigerante entre a entrada e a saída não deve ser maior que $3^{\circ} \mathrm{C}$. Valores elevados podem indicar uma alta temperatura na superfície do molde, que é o principal ponto que deve ser observado. Quando essa diferença é elevada, o projeto da refrigeração deve ser modificado. (DULEBA; GRESKOVIC,2012; REES, 1995; STEIKO, 2004).

Nos dois circuitos mostrados na figura 5, a diferença de temperatura do líquido refrigerante no sistema com canais convencionais para as duas cavidades foi aproximadamente $4^{\circ} \mathrm{C}$, já para o sistema com canais Conformal Cooling na cavidade superior foi aproximadamente $3^{\circ} \mathrm{C}$ e na cavidade inferior $1,5^{\circ} \mathrm{C}$.

Esses resultados mostram que a diferença de temperatura no circuito de refrigeração Conformal Cooling está de acordo com o limite especificado apresentando menores variações de temperatura, devido à transferência de calor ser mais eficiente, porque os canais estão mais próximos das cavidades do molde, em comparação com o circuito convencional. 
A eficiência do circuito de refrigeração influencia diretamente a qualidade e o tempo de resfriamento da peça. No mínimo 60\% dos defeitos visíveis encontrados em peças injetadas podem ser relacionados à ineficiência do sistema de refrigeração. (HUAMIN et al., 2008). A temperatura no molde ajuda a identificar quais regiões necessitam de maior remoção de calor.

A figura 6 apresenta a temperatura do molde na superfície em contato com o produto injetado (interface metal/plástico).

Figura 6: Regiões da peça com maior concentração de calor

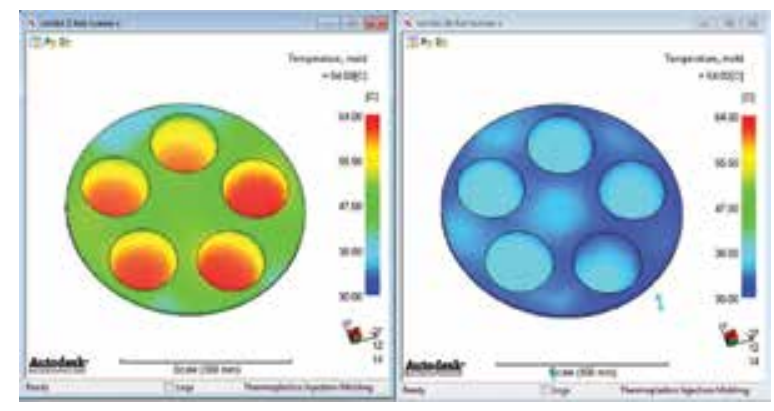

Fonte: Dos autores (2014)

Baseado na figura 6 é possível verificar que a temperatura mais elevada se encontra no interior das cinco cavidades do produto e que o circuito com Conformal Cooling apresenta elevada eficiência na transferência de calor quando comparado com o circuito convencional, pois quando o sistema atinge a etapa de ejeção da peça, as temperaturas encontradas no produto que foi resfriado com Conformal Cooling é de aproximadamente $22^{\circ} \mathrm{C}$ abaixo das temperaturas encontradas no produto resfriado com canais convencionais.

A figura 7 apresenta a temperatura no interior das cavidades superior e inferior do molde ao atingir a etapa de extração do produto.
Figura 7: Temperatura nas cavidades superior e inferior do molde

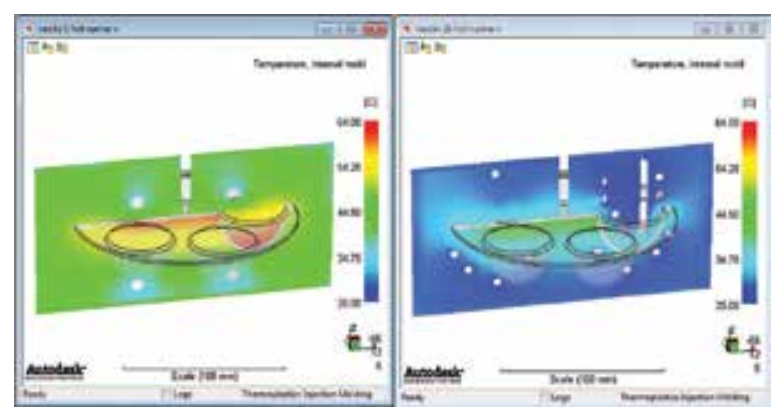

Fonte: Dos autores (2014)

A temperatura das cavidades está relacionada com a capacidade do sistema em resfriaro molde. Um bom desempenho requer uma temperatura homogênea, sem pontos quentes, que é a grande causa de tensões e empenamentos nas peças injetadas. Quando as cavidades são resfriadas homogeneamente com pequenas diferenças de temperatura, a possibilidade de defeito é baixa e a garantia de obter peças com qualidade será maior. (DULEBA; GRESKOVIC, 2012; REES, 1995; STEIKO, 2004).

Comparando os resultados obtidos nos circuitos apresentados na figura 7, pode ser observado que o circuito em Conformal Cooling tem maior eficiência e uniformidade no resfriamento do produto e o molde, apresentando algumas regiões com temperaturas próximas de $44^{\circ} \mathrm{C}$ (cor verde no gráfico) e na maioria das regiões das cavidades a temperatura está próxima de $30^{\circ} \mathrm{C}$ (cor azul no gráfico), considerando que as cavidades com sistema de refrigeração convencional possuem regiões com maior quantidade de temperaturas acima de $40^{\circ} \mathrm{C}$ (nas cores do gráfico: verde, amarelo e vermelho).

A figura 8 apresenta o tempo necessário para o sistema atingir a temperatura de ejeção do produto. 
Figura 8: Tempo de ciclo para que o sistema atinja a temperatura de ejeção do produto

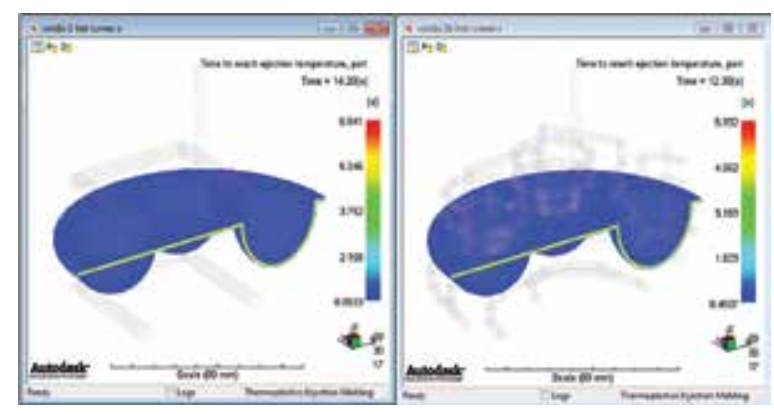

Fonte: Dos autores (2014)

Como é possível verificar na figura 8, o sistema que apresentou menor tempo de ciclo foi o circuito com canais em Conformal Cooling. A redução no tempo de ciclo dos circuitos com Conformal Cooling foi de aproximadamente 14\% comparado com o circuito convencional.

A velocidade de resfriamento do molde e da peça injetada (troca de calor) é um fator decisivo no desempenho econômico do molde de injeção, a maior velocidade e uniformidade no resfriamento do produto, o que resulta em produtos com melhores propriedades e menor custo agregado. (DULEBA;GRESKOVIC,2011; REES,1995; STEIKO,2004). A tabela 4 apresenta o resumo dos resultados das simulações dos sistemas com Conformal Cooling e convencional.

Tabela 4: Resumo dos resultados

\begin{tabular}{|l|c|c|}
\hline \multicolumn{1}{|c|}{ Canais de refrigeração } & Convencional & Conformal Cooling \\
\hline Taxa de fluxo (I/min) & & 1,5 \\
Cavidade superior & 3,2 & 2,8 \\
Cavidade inferior & 3,2 & \\
\hline Número de Reynolds & 10000 & 12000 \\
Cavidade superior & 10000 & 9000 \\
\hline Cavidade inferior & & $25,00-28,00$ \\
\hline Temperatura do refrigerante $\left({ }^{\circ} \mathrm{C}\right)$ & $25,00-29,00$ & $25,00-26,50$ \\
\hline Cavidade superior & $25,00-28,75$ & $30,00-42,00$ \\
\hline Cavidade inferior & $42,00-64,00$ & $30,00-40,00$ \\
\hline Temperatura do produto $\left({ }^{\circ} \mathrm{C}\right)$ & $35,00-60,00$ & 12,30 \\
\hline Temperatura do inserto $\left({ }^{\circ} \mathrm{C}\right)$ & 14,20 & \\
\hline Tempo de ciclo $(\mathbf{s})$ & & \\
\hline
\end{tabular}

Fonte: Dos autores (2014) 


\section{CONCLUSÕES}

De acordo com as simulações, o sistema de refrigeração com Conformal Cooling apresentou ter eficiência superior ao sistema convencional em vários pontos analisados:

- a temperatura do líquido refrigerante entre a entrada e a saída do sistema apresentou menor variação, mostrando ser um circuito mais homogêneo e eficiente;

- a temperatura do produto, ao atingir o tempo de ejeção, apresentou diminuição de $22^{\circ} \mathrm{C}$ nas regiões mais quentes;

- a temperatura do inserto, ao atingir o tempo de extração da peça, apresentou diminuição de $20^{\circ} \mathrm{C}$ nas regiões mais quentes;
- o tempo total de ciclo apresentou redução de $14 \%$.

Sendo assim, sugere-se que o sistema com Conformal Cooling é eficiente em moldes de injeção de polipropileno, pois permite o resfriamento uniforme do produto e do molde, o qual melhora significativamente as propriedades do produto injetado e mesmo na injeção de peças sem muita complexidade, como neste caso estudado, apresenta redução no tempo de refrigeração, o qual ao longo da vida útil do molde representará uma economia significante para as indústrias de transformação de plástico.

\section{AGRADECIMENTOS}

Os autores agradecem ao FUMDES pelo apoio a este projeto de pesquisa e também aos parceiros ENISE/DIPI, UNISOCIESC e empresa SOKIT Plásticos. 


\section{ANALYSIS OF INFLUENCE OF CONFORMAL COOLING IN A MOULD FOR INJECTION OF PLASTIC COMPARED WITH CONVENTIONAL REFRIGERATION THROUGH SIMULATION OF HEAT}

\author{
TRANSFER
}

\begin{abstract}
In the process of injection of plastic parts, the improvement of product quality and the reduction of time of injection cycle is the focus in all phases of the design of an injection mould. During the injection process, the cooling phase has the most significant impact on the overall cycle time, in addition to directly influence the properties of the moulded parts. Therefore, the cooling of the parts must be as uniform and fast as possible. To achieve this goal, the cooling channels in the cavities should be designed to adapt to the geometry of the product. However, conventional methods for manufacturing cooling channels allow only producing linear openings. Selective Laser Melting (SLM) is an Additive Manufacturing technology, based upon metalpowder, capable of producing parts layer by layer directly from the CAD model. It can be used for the manufacture of inserts for injection moulding of plastic with intricate cooling channels, known as Conformal Cooling. This study investigated, by simulation, the effect of Conformal Cooling during the injection moulding process to produce parts of polypropylene. Upper and lower insert were designed for the same product with a conventional cooling system and Conformal Coolingsystem. The results indicate that the Conformal Cooling in comparison with the conventional cooling system can supply uniform heat transfer during the cooling process and provide a reduction in duty cycle total time of approximately 14\%.
\end{abstract}

\footnotetext{
KEYWORDS: Simulation.

Injection of Plastic.

Conformal Cooling. Additive

manufacturing. SLM -

Selective Laser Melting.
}

\section{REFERÊNCIAS}

BRASKEM. Polipropileno H 201. Folha de dados - Revisão 6, Abril 2009.

Brito, A. M. et al. Manual do Projetista para Moldes de Injeção de Plásticos. V. 6, Sistemas de Alimentação e Escape de Gases. Marinha Grande, Brazil, Centimfe, 2004.

Dalgarno, K. W.; Stewart, T. D. Manufacture of Production Injection Mould Tooling Incorporating Conformal Cooling Channels via Indirect Selective Laser Sintering. Proceedings of the Institution of Mechanical Engineers, Parte B: Journal of Engineering Manufacture, v. 215, 1323-1332, 2001.

Dimla, D. E. et al. Design and Optimisation of Conformal Cooling Channels in Injection Moulding Tools. Journal of Materials Processing Technology, v. 164-165, 1294-1300, 2005.

Duleba, B.; Greskovic, F. Conformal Cooling for Plastics Injection Moulding. Strojar, 1-5, ISSN 1338-0761, 2012.

Harada, J. Moldes para Injeção de Termoplásticos - Projetos e Princípios Básicos. São Paulo, Brazil: Artiber, 2004.

Hsu, F. H.; Wang, H. C.; Huang, C. T.; Chang, R. Y. Investigation on conformal cooling system design in injection molding. 5th International Conference PMI, Ghent, Bélgica, 12 - 14 September, 2012.

Huamin, Z.; Bo, Y.; Yun, Z. 3D filling simulation of injection molding. Journal of Materials Processing Technology, v. 204, Iss. 1-3, 475-480, 2008.

Ilyas, I.; Taylor, C.; Dalgarno, K.; Gosden, J. Design and manufacture of injection mould tool inserts produced using indirect SLS and machining processes. Rapid Prototyping Journal, v. 16, Iss: 6, 429-440, 2010.

Li, C. L.; Li, C.G.; Mok, A. C. K. Automatic layout design of plastic injection mould cooling system. Computer-Aided Design. v. 37, 645-662, 2005.

Manrich, S. Processamento de termoplásticos: rosca única, extrusão e matrizes, injeção e moldes. São Paulo: Artiber, 2005.

Park, H. S.; Dang, X. P. Design and simulationbased optimization of cooling channels for plastic injection mold, New technologies: trends, innovations and research, Prof. Volosencu, C. (Ed.), Isbn: 978-953-51-0480-3, InTech, 19-44, 2012. 
Rees, H. Mold Engineering. Munich; Vienna; New York: Hanser; Cincinnati: Hanser/Gardner, 1995.

Sacchelli, C. M.; Cardoso, C. A. 2013. Análise da simulação de Refrigeração no processo de injeção de termoplásticos. Portal do Molde. Disponível em: <http://www.moldesinjecaoplasticos.com.br/ refrigeração.asp >. Acesso em: 10 abr. 2013.

Sant'Anna, J. A. P.; Wiebeck, H. Ferramentas para seleção de materiais poliméricos buscando substituir materiais metálicos. $17^{\circ} \mathrm{CBECIMat}$ Congresso Brasileiro de Engenharia e Ciência dos Materiais, Foz do Iguaçu, Brasil, 15-19 Novembro, 2006.

Siegfried, M. 2009. Optimized mould temperature control procedure using DMLS, EOS whitepaper. Eureka the site for engineering design.

Silva, S. L. 2009. Sistemática para o projeto do sistema de refrigeração de moldes para injeção de polímeros. Dissertação apresentada à Universidade Federal de Santa Catarina - UFSC, Florianópolis, Brasil.

Souza, A. F.; Bodziak, S. Advanced Free Form Manufacturing by Computer Aided Systems Cax, Mechanical Engineering, Dr. Murat Gokcek (Ed.), ISBN: 978-953-51-0505-3, InTech, 2012.

Steiko, W. Avaliação do projeto térmico do molde garante qualidade e redução de custos. Plástico Industrial, v. 1, 64-71, Novembro, 2004.

Venerus, D. C., et al. Relaxation of Anisotropic Thermal Diffusivity in a Polymer Melt Following Step Shear Strain. The American Physical Society, v. 82, N. 2, 366-369, ISSN 0031-9007, 1999.

Wohlers, T. 2012. Wohlers Report: Additive manufacturing and $3 \mathrm{D}$ printing state of the industry. Annual world wide progress report, Fort Collins, Wohlers Associates, 287, 2012.

Yadroitsev, I.; Smurov, I. Selective laser melting technology: from the single laser melted track stability to 3D parts of complex shape. Physics Procedia, v. 5, 551 - 560, 2010.

Yadroitsev, I.; Smurov, I. Surface morphology in selective laser melting of metal powders. Physics Procedia. v. 12, $264-270,2011$.

\section{SOBRE OS AUTORES}

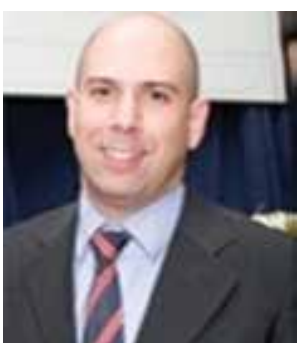

Adriano Fagali de

Souza

Doutor em Engenharia

Mecânica pela

Universidade de São Paulo

EESC USP (2004); é mes-

tre em Engenharia de Produção e engenheiro de Produção Mecânica pela Universidade Metodista de Piracicaba. Em 1992 concluiu o curso técnico em mecânica pelo Colégio Técnico da UNICAMP. É professor da Universidade Federal de Santa Catarina - UFSC/Joinville. Atua e coordena diversos projetos de pesquisa, em temas relativos à fabricação de produtos contendo formas complexas, empregando a cadeia CAD/CAM/CNC - CAx - fabricação de moldes e matrizes. Estuda também a Tecnologia de Usinagem em Altas Velocidades (High Speed Cutting-HSC).Élíder do Grupo de Pesquisa em Manufatura Auxiliada por Computador - GPCAM. É autor dos livros: Manufacturing Complex Geometries using High Speed Cutting Technology, pela editora VDM Verlag,2010,e Engenharia Integrada por Computador e sistemas CAD/CAM/CNC, pela editora Artliber (2a edição em 2013). 


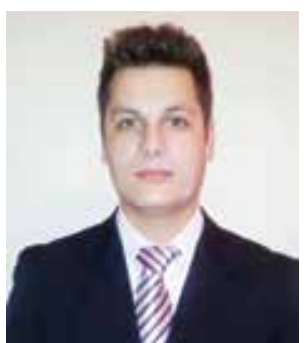

Rafael Felipe Fronza

\section{Santos}

É aluno do curso de

Engenharia Mecânica pelo

Centro Universitário Tupy

-UNISOCIESC.Em 2013

foi bolsista de Iniciação Científica CNPq-PIBIC desenvolvendo pesquisa na área de simulação de injeção para moldes com canais conformal cooling pela UNISOCIESC. Atualmente é bolsista na empresa RezekEngenharia, desenvolvendo projetos estruturais de andaimes, elevadores e galpões de estrutura metálica.

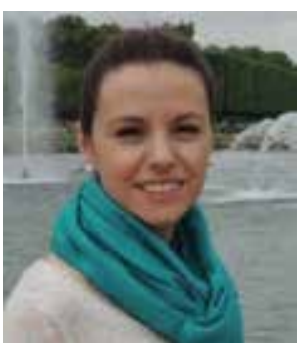

Sabrina Marques

Mestre em Engenharia Mecânica (2014) e EngenheiraMecânica(2011) pelo Centro Universitário Tupy-UNISOCIESC.Em 2006 concluiu o curso técnico em Mecânica pela Escola Técnica Tupy - ETT. Em 2013 realizou intercâmbio entre os laboratórios DIPI da École Nationale d'Ingénieurs de Saint-Etienne - ENISE/ França e Promolde da UNISOCIESC, desenvolvendo pesquisas em Fusão Seletiva a Laser para a fabricação de peças metálicas com maior densidade. Atualmente é aluna em regime especial do curso de Doutorado em Engenharia de Materiais pela Universidade do Estado de Santa Catarina UDESC.É pesquisadora na área de Fusão Seletiva a Laser (Selective Laser Melting-SLM) em diferentes ligas metálicas no Instituto SENAI de Inovação em Sistemas de Manufatura. 